\title{
CLASSIFICAÇÃO INTERNACIONAL DE MOTIVOS DE CONSULTA PARA ASSISTÊNCIẢ PRIMÁRIA: TESTE EM ALGUMAS ÁREAS BRASILEIRAS
}

\author{
Maria Lúcia Lebrão*
}

\begin{abstract}
LEBRÃO, M. L. Classificação intemacional de motivos de consulta para assistência primária:
\end{abstract} teste em algumas áreas brasileiras. Rev. Saúde públ, S. Paulo, 19: 69-78, 1985.

RESUMO: E apresentada uma nova classificação internacional de motivos de consulta em assistência primária proposta pela OMS, comentando os resultados de teste realizado em seis locais do Brasil, para verificação da abrangência e exequibibilidade da nova classificação. Os re. sultados foram satisfatórios, levando à conclusāo da viabilidade do uso do instrumento.

UNITERMOS: Classificação internacional para assistência primária.

\section{INTRODUÇÃO}

O processo de tomada de decisáo, em qualquer área do planejamento, tem como base a informação. A saúde não pode, portanto, fugir a essa regra. Acresce-se a isso a enorme discussão que se verifica nos últimos anos a respeito da necessidade de se estabele. cerem novos padrões de política de saúde, pensando-se, principalmente, em assistência primária. Nessa área, constitui aspecto relevante a questão da existência de uma conexão entre a razão da entrada de uma pessoa no sistema de saúde, vindo a se tornar um paciente, e o que acontece a essa pessoa depois da sua entrada. Com essa preocupação, a Organização Mundial da Saúde formou um grupo de especialistas que, a partir de novembro de 1978 , iniciou as discussóes para a elaboração de uma nova classificação que auxilie na resposta à questão: porque uma pessoa procura um serviço de saúde? ${ }^{8}$

A maioria das classificaçðes de doenças tem por objetivo classificar a interpretação pelo "provedor" da assistência de saúde, da doença, afecção ou traumatismo do doente. $\mathrm{Na}$ "Classificação Internacional para Assistên. cia Primária" (CIAP) são classificadas as razões que levaram as pessoas a pedir assistência, vistas da perspectiva do paciente. $A$ informação que se classifica é a que se obtém no início do contato do provedor com o pa. ciente, antes que se disponha de informação suficiente para a formulação do diagnósti$\mathrm{co}^{4}$. Trata-se, portanto, de uma classificação orientada para o paciente mais que para a doença ou para o provedor da assistência à Saúde 4 . Além da doença, muitos outros problemas podem levar as pessoas a visitar um serviço de assistência primária: problemas econômicos, sociais e psicológicos, ca. rências, ansiedade, medos, incertezas. Tudo isso junto se traduz nos "problemas das pessoas". Por essa razão, o grupo de trabalho sentiu-se livre para aceitar qualquer plano, conceito ou sugestão que preenchesse os novos propósitos, não se sentindo restrito à Classificação Internacional de Doenças, 9a

- Do Departamento de Epidemiologia da Faculdade de Saúde Pública da Universidade de Sáo Paulo Av. Dr. Arnaldo, 715 - 01255 - Sáo Paulo, SP - Brasil. 
LEBRÃO, M.L. Classificação internacional de motivos de consulta para assistência primária: teste em algumas áreas brasileiras. Rev. Saride puibl., S. Paulo, 19:69-78, 1985.

Revisão (CID-9) ${ }^{3,8}$.

A razão básica da não utilização completa da CID-9 foi ser a mortalidade o seu referencial primeiro e, também, a falta de detalhes na área de queixas e sintomas, aspectos importantes na assistência primária. Porém, a CID-9 serviu de base para uma das partes da classificação: o componente 7, Diagnósticos e Doenças. Os títulos de categoria que fi. guram no componente 7 sáo os mesmos da "International Classification of Health Pro. blems in Primary Care" (ICHPPC-2) ${ }^{2}$, que é uma versão da CID.9 modificada para a atenção primária de saúde. Outras classificaçðes existentes, como a elaborada pelo North American Primary Care Research Group (NAPCRG-1): "A process code for primary care"s e a do National Ambulatory Medical Care Survey: "Reason for visit (NAMCS -RFV)", 7 , não satisfaziam completamente, da mesma forma. Dessa maneira, decidiu-se que seria mais vantajoso incorporar o melhor de cada sistema já existente. Assim surgiu a primeira versão da "Reasons for Encounter Classification (RFE-C)" traduzida e publicada pelo Centro da OMS para Classificação de Doenças em Português sob o título de "Classificação de Motivos de Consulta" (CMC) ${ }^{1}$ que, após os testes, foi substituida pela última versão, aqui apresentada (CIAP).

\section{A CLASSIFICAÇÃO INTERNACIONAL PARA ASSISTENCIA PRIMẢRIA}

A classificação proposta pelo grupo da OMS está estruturada em dois eixos, como pode ser visto na Figura: capítulos e componentes. Os capítulos são em número de 17, a maioria abrangendo os sistemas orgâ. nicos (B, D, F, H, K, L, N, R, S, T, U, X, Y); os demais não obedecem a critérios anatômicos, mas correspondem a "problemas gerais" (capítulo A), "psicológicos" (P), "relativos à reprodução" (W) e "sociais" (Z). As doenças infecciosas, os tumores, os traumatismos e as anomalias congênitas não constituem capítu. los independentes como na Classificação Internacional de Doenças mas, acham-se representados no componente Diagnóstico/Doença de cada capítulo, que por sua vez é repre- sentado por uma letra que é o primeiro símbolo que figura em todos os códigos e rubricas que estão contidos nos capítulos. Estes, por sua vez, estão subdivididos nos mesmos sete componentes. Cada componente compreende uma série de códigos de dois algarismos, colocados após a letra de código correspondente ao capítulo. Os componentes estão distribuídos da seguinte maneira:

\section{Componente 1: Sintomas e queixas} códigos de 01 a 29

Componente 2: Procedimentos diagnósticos e preventivos

códigos de 30 a 49

Componente 3: Medicação, tratamento e procedimentos terapêtuticos

códigos de 50 a 59

Componente 4: Resultados codigos 60 e 61

Componente 5: Razós Administrativas código 62

Componente 6: Encaminhamentos e outras códigos de 63 a 69 razóes para encontro

Componente 7: Diagnósticos/Doenças códigos de 70 a 99

Este componente 7 so pode ser usado quando o paciente expressa o motivo da procura em termos de um diagnóstico.

Aplica-se, pois, um sistema relativamente simples de classificação biaxial com 3 caracteres, baseado em sete componentes fixos, cinco dos quais (de 2 a 6) têm, em todos os capítulos, códigos de 2 algarismos análogos. Como exemplo, pode-se citar:

A03 Febre.......... componente 1, sintomas e queixas do capitulo A - Problemas gerais $e$ inespecíficos.

D41 RX de estômago. . componente 2, procedimentos diagnósticos e preventivos do capitulo D. Aparelho Digestivo.

Y82 Hipospádia . . . . . componente 7, Diag. nósticos/Doenças do 
LEBRÃO, M.L. Classificação internacional de motivos de consulta para assistência primária: teste em algumas áreas brasileiras. Rev. Saúde puibl., S. Paulo, 19: 69-78, 1985.

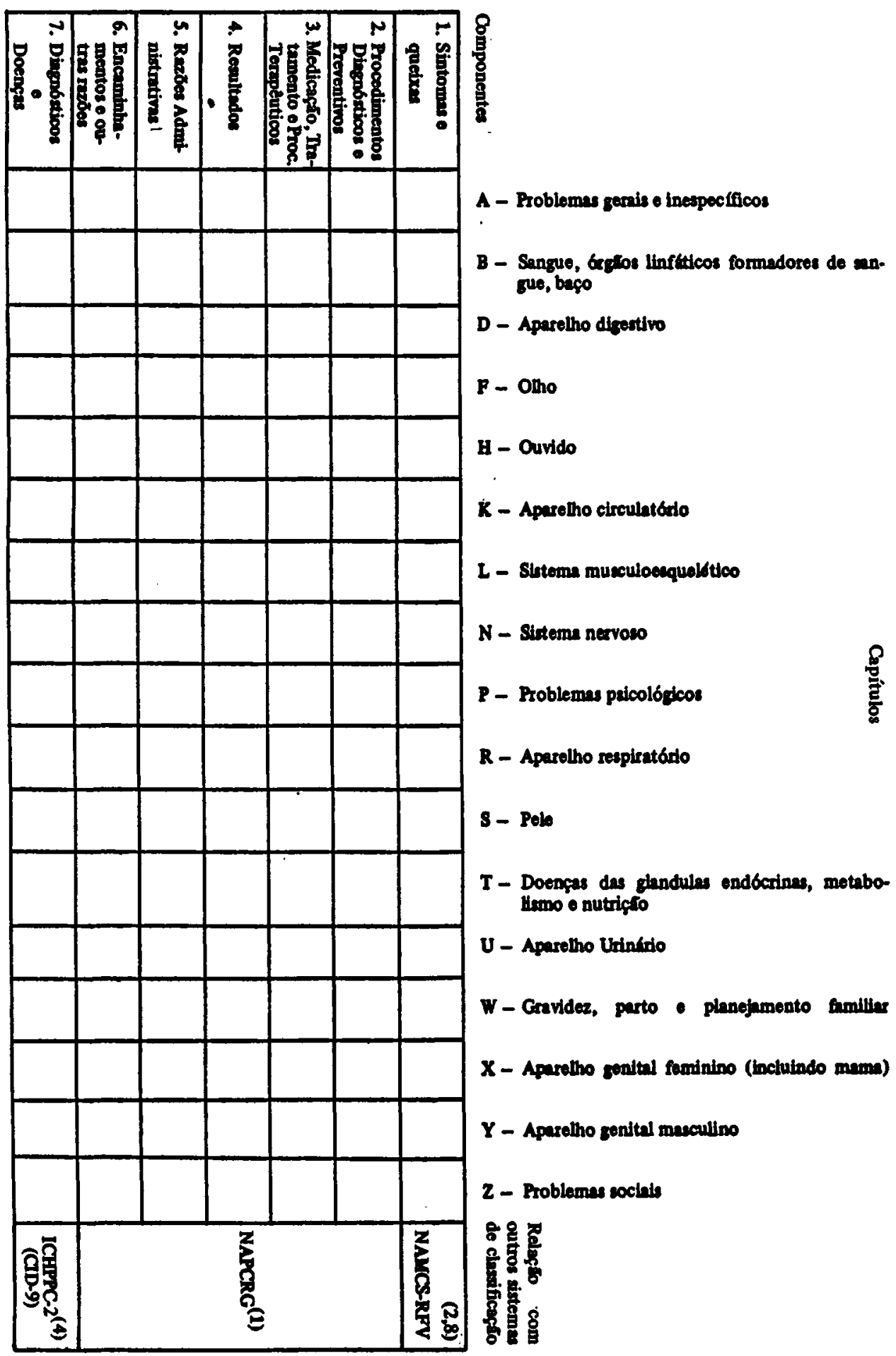

Figura - Estrutura da Classificação Internacional para Assistência Primária. 
LEBRÃO, M.L. Classificação internacional de motivos de consulta para assistência primária: teste em algumas áreas brasileiras. Rev. Saúde pribl., S. Paulo, 19: 69-78, 1985.

capítulo Y - Aparetho genital masculino.

O esquema apî́esentado na Figura dá uma idéia da estrutura da classificação que está sendo proposta para a assistência primá. ria.

\section{TESTES DA CLASSIFICAÇÃO}

Visando a avaliar a exeqüibilidade do uso do modelo proposto, em 1980 foi realizado um estudo piloto nos Países Baixos. À vista dos resultados obtidos foi empreendido, em 1983, um novo teste de viabilidade em outros países: Austrália, Barbados, Estados Unidos da América, Hungria, Malásia, Noruega, Países Baixos e Brasil. Entre nós, esse teste foi realizado em seis locais: (1) Pronto Atendimento do Instituto da Criança do Hospital das Clínicas de Sáo Paulo, SP; (2) Centro de Saúde Escola Dr. Samuel B. Pessoa (Butantã), SP; (3) Hospital Centro de Saúde de Cotia, Sáo Paulo;(4) Serviço Especial de Saúde de Araraquara, Sáo Paulo; (5) Centro de Saúde - Escola da Faculdade de Medicina de Botucatu, SP; (6) Centros de Saúde do Departamento de Medicina Social da Faculdade de Medicina da Universidade Federal de Pelotas, RS. No total foram utilizados 21 pesquisadores, entre médicos residentes e funcionários, sob a coordenação de um pesquisador principal. Como se tratava de um teste de classificação, não interessando, no momento, o conhecimento da verdadeira morbidade dos locais estudados, não houve preocupaçōes com a representatividade do material analisado. Assim, participaram dessa fase do estudo aqueles grupos que se mostraram interessados e que tinham facilidades para a realização do trabalho. Foram analisados 11.186 encontros (assim chamado o número de vêzes que os pacientes procuraram qualquer dos serviços incluídos no estudo) que resultaram em 16.271 razós para a procura dos serviços.

Os motivos de consulta foram registrados em folha pré-codificada, encaminhada para a Organização Mundial de Saúde, em Genebra, centralizadora dos dados, que os passava para o computador. Um problema surgido foi decorrente do fato de essas informaçðes terem sido registradas em português e lidas por alguém de língua inglesa. Isso exigiu, dos participantes, o registro dos motivos de consulta, em letra de forma, e, da coordenação, uma revisão de todos os casos enviados. Alguns participantes tinham caligrafia de leitura bastante difícil para pessoas que desconheciam a língua portuguesa. Nesses casos, os dados foram digitados e enviados em folhas de computação. Isso permitiu que toda a informação enviada do Brasil fosse apro. veitada.

A utilização da classificação é bastante simples, o que facilitou o treinamento dos participantes. Foi gasto, em média, um período de 4 horas com cada grupo (cada localidade) para o treinamento inicial. Após o início da aplicação foram feitas visitas pe. riódicas para discussão das dúvidas. Todos os questionamentos e sugestoes surgidas durante o trabalho foram encaminhados, ao final, para o grupo responsável pela classificação (OMS) e foram aproveitados na reformulação da classificação, resultando na última revisão, que é a apresentada no presente trabalho.

\section{RESULTADOS E COMENTÁRIOS}

Alguns resultados interessantes podem ser verificados na análise do teste realizado no Brasil. Das 16.271 razões apresentadas para a prooura de um serviço de saúde, às do capítulo A (Problemas Gerais e inespecificos) foram as mais referidas $(28,5 \%)$, seguidas pelas do Aparelho Respiratório (20,6\%) e Aparelho Digestivo (16,3\%). A distribuição da frequeência dos demais capítulos pode ser vista na Tabela 1. Quanto às razóes apresentadas, em relação aos com. ponentes, $70,8 \%$ se manifestaram por sinto. mas ou queixas (componente 1 - Figura) tendo os restantes $29,2 \%$ se distribuído entre os outros seis (Tabela 2), sendo referidos todos os capítulos. Ainda na Tabela 1 pode-se ver que $75,2 \%$ das queixas foram apresentadas pela primeira vez naquele serviço, sendo apenas $24,8 \%$ por seguimento. $\mathbf{E}$ interes- 
LEBRÃO, M.L. Cassificaçð̃o internacional de motivos de consulta para assistência primária: teste em algumas áreas brasileiras. Rev. Sauide públ., S. Paulo, 19: 69-78, 1985.

\section{TA B E L A 1}

Distribuiçæo dos motivos de consulta segundo os tipos de consulta (nova ou retomo) em vários locais de atendimento, Brasil, em 1983.

\begin{tabular}{|c|c|c|c|c|c|c|}
\hline \multirow[b]{3}{*}{ Capitulos* } & \multicolumn{4}{|c|}{ Tipo de Consulta } & \multirow{2}{*}{\multicolumn{2}{|c|}{ Total }} \\
\hline & \multicolumn{2}{|c|}{ 19 (nova) } & \multicolumn{2}{|c|}{ Retorno } & & \\
\hline & Nọ & $\%$ & No & 9 & Nọ & \% \\
\hline $\mathbf{A}$ & $\begin{array}{c}3084 \\
(25,2)\end{array}$ & 66,6 & $\begin{array}{c}1552 \\
(38,5)\end{array}$ & 33,4 & $\begin{array}{l}4634 \\
(28,5)\end{array}$ & 100,0 \\
\hline B & $\begin{array}{c}234 \\
(1,9)\end{array}$ & 82,7 & $\begin{array}{c}49 \\
(1,2)\end{array}$ & 17,3 & $\begin{array}{c}283 \\
(1,7)\end{array}$ & 100,0 \\
\hline D & $\begin{array}{l}2236 \\
(18,3)\end{array}$ & 84,2 & $\begin{array}{c}421 \\
(10,4)\end{array}$ & 15,8 & $\begin{array}{l}2657 \\
(16,3)\end{array}$ & 100,0 \\
\hline $\mathbf{F}$ & $\begin{array}{c}171 \\
(1,4)\end{array}$ & 89,5 & $\begin{array}{c}20 \\
(0,5)\end{array}$ & 10,5 & $\begin{array}{l}191 \\
(1,2)\end{array}$ & 100,0 \\
\hline $\mathbf{H}$ & $\begin{array}{c}366 \\
(3,0)\end{array}$ & 83,9 & $\begin{array}{c}70 \\
(1,7)\end{array}$ & 16,1 & $\begin{array}{l}436 \\
(2,7)\end{array}$ & 100,0 \\
\hline $\mathbf{K}$ & $\begin{array}{l}167 \\
(1,4)\end{array}$ & 45,1 & $\begin{array}{l}203 \\
(5,0)\end{array}$ & 54,9 & $\begin{array}{l}370 \\
(2,3)\end{array}$ & 100,0 \\
\hline $\mathbf{L}$ & $\begin{array}{c}586 \\
(4,8)\end{array}$ & 85,7 & $\begin{array}{c}98 \\
(2,4)\end{array}$ & 14,3 & $\begin{array}{l}684 \\
(4,2)\end{array}$ & 100,0 \\
\hline $\mathbf{N}$ & $\begin{array}{c}466 \\
(3,8)\end{array}$ & 80,9 & $\begin{array}{l}110 \\
(2,7)\end{array}$ & 19,1 & $\begin{array}{l}576 \\
(3,5)\end{array}$ & 100,0 \\
\hline $\mathbf{P}$ & $\begin{array}{c}235 \\
(1,9)\end{array}$ & 73,7 & $\begin{array}{c}84 \\
(2,1)\end{array}$ & 26,3 & $\begin{array}{l}319 \\
(2,0)\end{array}$ & 100,0 \\
\hline $\mathbf{R}$ & $\begin{array}{c}2665 \\
(21,8)\end{array}$ & 79,5 & $\begin{array}{c}688 \\
(17,0)\end{array}$ & 20,5 & $\begin{array}{l}3353 \\
(20,6)\end{array}$ & 100,0 \\
\hline $\mathbf{S}$ & $\begin{array}{l}848 \\
(6,9)\end{array}$ & 83,7 & $\begin{array}{l}165 \\
(4,1)\end{array}$ & 16,3 & $\begin{array}{c}1013 \\
(6,2)\end{array}$ & 100,0 \\
\hline $\mathbf{T}$ & $\begin{array}{c}87 \\
(0,7)\end{array}$ & 60,4 & $\begin{array}{c}57 \\
(1,4)\end{array}$ & 39,6 & $\begin{array}{l}144 \\
(0,9)\end{array}$ & 100,0 \\
\hline $\mathrm{U}$ & $\begin{array}{c}210 \\
(1,7)\end{array}$ & 74,7 & $\begin{array}{c}71 \\
(1,8)\end{array}$ & 25,3 & $\begin{array}{l}281 \\
(1,7)\end{array}$ & 100,0 \\
\hline $\mathbf{X}$ & $\begin{array}{c}694 \\
(5,7)\end{array}$ & 67,9 & $\begin{array}{c}328 \\
(8,1)\end{array}$ & 32,1 & $\begin{array}{c}1022 \\
(6,3)\end{array}$ & 100,0 \\
\hline $\mathbf{Y}$ & $\begin{array}{c}71 \\
(0,6)\end{array}$ & 86,6 & $\begin{array}{c}11 \\
(0,3)\end{array}$ & 13,4 & $\begin{array}{c}82 \\
(0,5)\end{array}$ & 100,0 \\
\hline $\mathbf{Z}$ & $\begin{array}{c}111 \\
(0,9)\end{array}$ & 49,1 & $\begin{array}{l}115 \\
(2,8)\end{array}$ & 50,9 & $\begin{array}{l}226 \\
(1,4)\end{array}$ & 100,0 \\
\hline Total & $\begin{array}{r}231 \\
(100,0)\end{array}$ & 75,2 & $\begin{array}{r}4042 \\
(100,0)\end{array}$ & 24,8 & $\begin{array}{l}16271 \\
(100,0)\end{array}$ & 100,0 \\
\hline
\end{tabular}

* O capítulo W náo está inclúdo nesta Tabela por ter sido acrescentado após a reformulaçto. 
LEBRĀO, M.L. Classificaḉo internacional de motivos de consulta para assistência prim ́ria: teste em algumas áreas brasileiras. Rev. Saúde públ., S. Paulo, 19: 69-78, 1985.

\section{T A B E L A 2}

Distribuiçáo dos motivos de consulta segundo os componentes da Classificação Internacional para Assistência Primária, em vários locais de atendimento, Brasil, em 1983. (NQ e \%)

\begin{tabular}{crr}
\hline Componentes & No & \multicolumn{1}{c}{$\%$} \\
\hline 1 & 11513 & 70,7 \\
2 & 1576 & 9,8 \\
3 & 338 & 2,1 \\
4 & 679 & 4,2 \\
5 & 260 & 1,6 \\
6 & 705 & 4,3 \\
7 & 1180 & 7,3 \\
\hline Total & 16271 & 100,0 \\
\hline
\end{tabular}

sante notar que nos capítulos $\mathrm{K}$ (Aparelho Circulatório) e $Z$ (Razóes Sociais) foi maior a quantidade de retornos.

Em relaçăo ao sexo, a Tabela 3 mostra que foi maior a proporção de mulheres, como é comum ser encontrado nos atendimentos de saúde $(56,9 \%$ para $43,1 \%$ do sexo masculino).

$\mathrm{Na}$ Tabela 4 pode ser vista a distribuição dos motivos de consulta segundo os grupos etários. De uma maneira geral, os grupos de idade seguem o mesmo padrão de queixas do global dos atendimentos, com ênfase nas queixas gerais e inespecíficas, aparelho respiratório e aparelho digestivo. Constituem exceção os grupos de 15 a 24 e de 25 a 44 anos por apresentarem como principais queixas aquelas do capitulo $X$ (Aparelho Genital Feminino). Também os pacientes de 45 anos e mais afastam-se um pouco da distribuição geral ao apresentarem as queixas referentes ao Aparelho Circulatório (K) como a segunda mais freqüente.

$O$ grande peso constituído pelos menores de 15 anos $(66,5 \%)$ reflete o maior número de serviços de pediatria utilizados, não se ignorando o fato de ser esse grupo um grande consumidor de serviços de saúde.

\section{CONCLUSÕES}

Com base na análise feita a partir do teste, foram sugeridas algumas modificaçoes na classificação, como no capítulo de Pele (S) que trazia dificuldades em virtude da diversidade de termos usados pelos pacientes. 0 componente 1 (Sintomas e Queixas), que tinha 13 possiveis códigos, passou a ter 27.

Alguns termos apresentaram problemas de bilocação, ou seja, podiam ser classificados em dois capitulos diferentes; exemplo disso era "dor nas pernas", que podia estar em A 16 e L 14. Na última versão, foi locali. zada no capitulo L - Sistema Músculo-Esquelético. Os códigos relativos a lesóes e traumatismos, que eram apenas 2 no capitu. lo $\mathrm{A}$, foram expandidos para 9 , na última verș̃o.

Outro problema sentido e que pôde ser resolvido foi o relativo às mudanças das características das fezes. Os pacientes têm uma enorme variedade de termos para descrever queixas e sintomas relativos ds fezes e a primeira versăo da classificação era bastante restrita a esse respeito. Com a expansáo do capítulo, esses termos foram incluídos.

Assim como essas questoes, outras menores puderam ser resolvidas.

Ao apresentar os resultados e comentários pretendeu-se dar idéia geral do que foi encontrado nessa fase de teste do instrumento. $O$ próximo passo será avaliar o uso dessa classificação na prática da assistência primá. ria, no planejamento em saude e em pesquisa. A utilizaçăo dessa classificaçăo năo deve ficar restrita aos médicos, mas deve ser aceitável para enfermeiros, agentes de saúde, visitadores sanitários e todo o pessoal paramédico trabalhando em serviços de assistência primária. 
LEBRÃO, M.L. Classificaço internacional de motivos de consulta para assiatóncia primária: teste em algumes áreas brasileiras. Rev. Srúde públ., S. Paulo, 19:69-78, 1985.

TAB ELA 3

Distribuigéo dos motivos de consulta segundo sexo e capítulos da Classiflcaçón Internacional para Assistência Primária, em vários locais de atendimento, Brasil, em 1983 (No e \%)

\begin{tabular}{|c|c|c|c|c|c|c|}
\hline \multirow[b]{3}{*}{ Capítulos } & \multicolumn{4}{|c|}{ Sexo } & \multirow{3}{*}{ Total } & \\
\hline & \multicolumn{2}{|c|}{ Masculino } & \multicolumn{2}{|c|}{ Feminino } & & \\
\hline & No & $x$ & Nọ & $\%$ & & \\
\hline $\mathbf{A}$ & $\begin{array}{l}2163 \\
(30,9)\end{array}$ & 46,7 & $\begin{array}{c}2469 \\
(26,6)\end{array}$ & 53,3 & $\begin{array}{l}4634 \\
(28,5)\end{array}$ & 100,0 \\
\hline B & $\begin{array}{c}126 \\
(1,8)\end{array}$ & 44,5 & $\begin{array}{l}157 \\
(1,7)\end{array}$ & 55,5 & $\begin{array}{l}283 \\
(1,7)\end{array}$ & 100,0 \\
\hline D & $\begin{array}{c}1260 \\
(18,0)\end{array}$ & 47,4 & $\begin{array}{l}1397 \\
(15,1)\end{array}$ & 52,6 & $\begin{array}{l}2657 \\
(16,3)\end{array}$ & 100,0 \\
\hline $\mathbf{F}$ & $\begin{array}{c}90 \\
(1,3)\end{array}$ & 47,1 & $\begin{array}{l}101 \\
(1,1)\end{array}$ & 52,9 & $\begin{array}{c}191 \\
(1,2)\end{array}$ & 100,0 \\
\hline $\mathbf{H}$ & $\begin{array}{c}226 \\
(3,2)\end{array}$ & 51,8 & $\begin{array}{l}210 \\
(2,3)\end{array}$ & 48,2 & $\begin{array}{l}436 \\
(2,7)\end{array}$ & 100,0 \\
\hline $\mathbf{K}$ & $\begin{array}{c}123 \\
(1,8)\end{array}$ & 33,2 & $\begin{array}{l}247 \\
(2,7)\end{array}$ & 66,8 & $\begin{array}{l}370 \\
(2,3)\end{array}$ & 100,0 \\
\hline $\mathbf{L}$ & $\begin{array}{l}281 \\
(4,0)\end{array}$ & 41,1 & $\begin{array}{l}403 \\
(4,4)\end{array}$ & 58,9 & $\begin{array}{c}684 \\
(4,2)\end{array}$ & 100,0 \\
\hline $\mathbf{N}$ & $\begin{array}{l}169 \\
(2,4)\end{array}$ & 29,3 & $\begin{array}{l}407 \\
(4,4)\end{array}$ & 70,7 & $\begin{array}{l}576 \\
(3,5)\end{array}$ & 100,0 \\
\hline $\mathbf{P}$ & $\begin{array}{l}126 \\
(1,8)\end{array}$ & 39,5 & $\begin{array}{l}193 \\
(2,1)\end{array}$ & 60,5 & $\begin{array}{l}319 \\
(2,0)\end{array}$ & 100,0 \\
\hline $\mathbf{R}$ & $\begin{array}{c}1623 \\
(23,1)\end{array}$ & 48,4 & $\begin{array}{l}1730 \\
(18,7)\end{array}$ & 51,6 & $\begin{array}{l}3353 \\
(20,6)\end{array}$ & 100,0 \\
\hline $\mathbf{S}$ & $\begin{array}{l}484 \\
(6,9)\end{array}$ & 47,8 & $\begin{array}{l}529 \\
(5,7)\end{array}$ & 52,2 & $\begin{array}{c}1013 \\
(6,2)\end{array}$ & 100,0 \\
\hline$T$ & $\begin{array}{c}59 \\
(0,8)\end{array}$ & 40,9 & $\begin{array}{c}85 \\
(0,9)\end{array}$ & 59,1 & $\begin{array}{c}144 \\
(0,9)\end{array}$ & 100,0 \\
\hline $\mathbf{U}$ & $\begin{array}{c}82 \\
(1,2)\end{array}$ & 29,2 & $\begin{array}{l}199 \\
(2,1)\end{array}$ & 70,8 & $\begin{array}{l}281 \\
(1,7)\end{array}$ & 100,0 \\
\hline $\mathbf{X}$ & $\overline{(-)}$ & - & $\begin{array}{l}1022 \\
(11,0)\end{array}$ & 100,0 & $\begin{array}{c}1022 \\
(6,3)\end{array}$ & 100,0 \\
\hline $\mathbf{Y}$ & $\begin{array}{c}82 \\
(1,2)\end{array}$ & 100,0 & $\overline{(-)}$ & - & $\begin{array}{c}82 \\
(0,5)\end{array}$ & 100,0 \\
\hline $\mathbf{Z}$ & $\begin{array}{l}112 \\
(1,6)\end{array}$ & 49,6 & $\begin{array}{l}114 \\
(1,2)\end{array}$ & 50,4 & $\begin{array}{l}226 \\
(1,4)\end{array}$ & 100,0 \\
\hline Total & $\begin{array}{c}7013 \\
(100,0)\end{array}$ & 43,1 & $\begin{array}{c}9257 \\
(100,0)\end{array}$ & 56,9 & $\begin{array}{l}16271 \\
(100,0)\end{array}$ & 100,0 \\
\hline
\end{tabular}




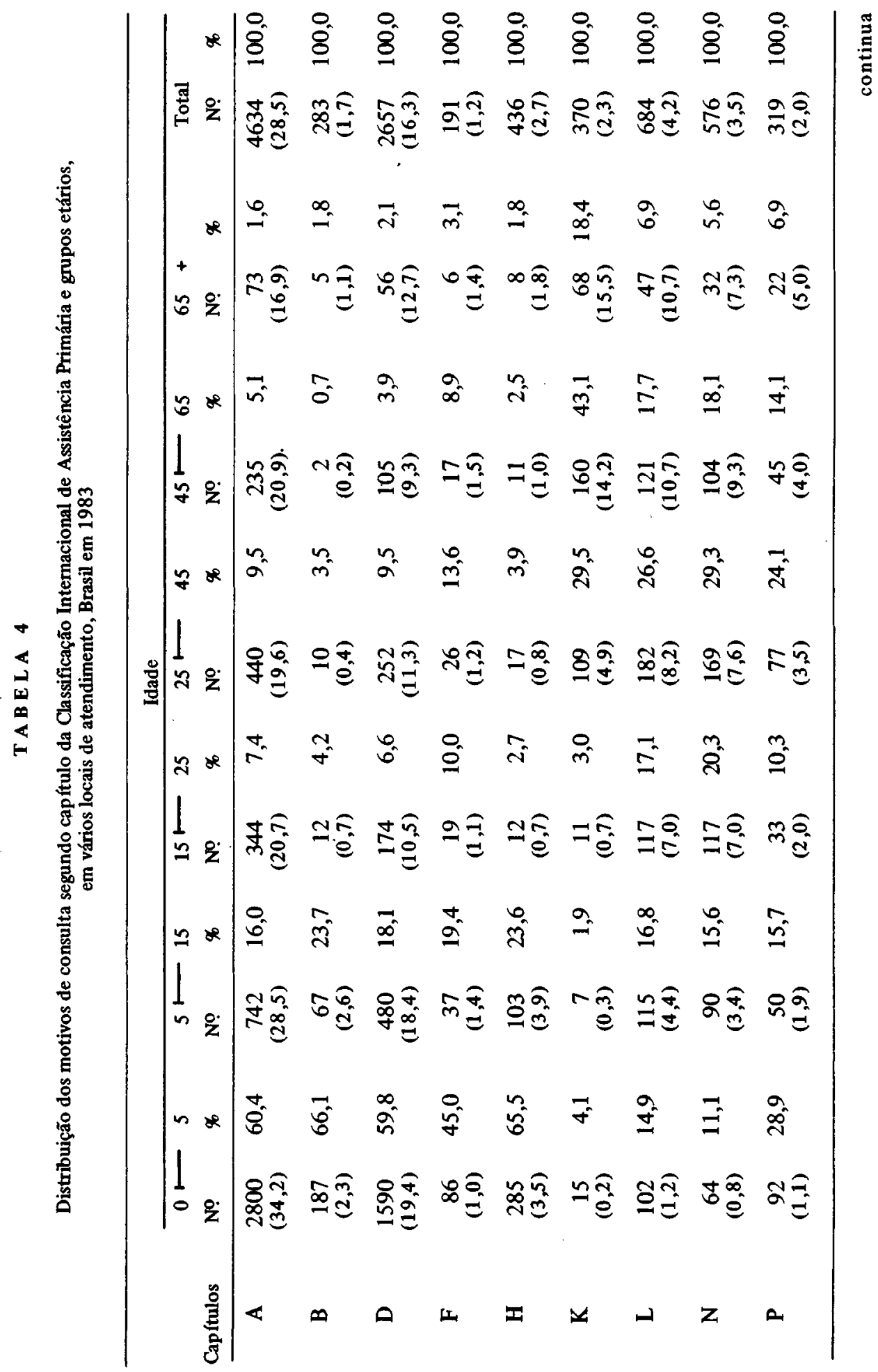




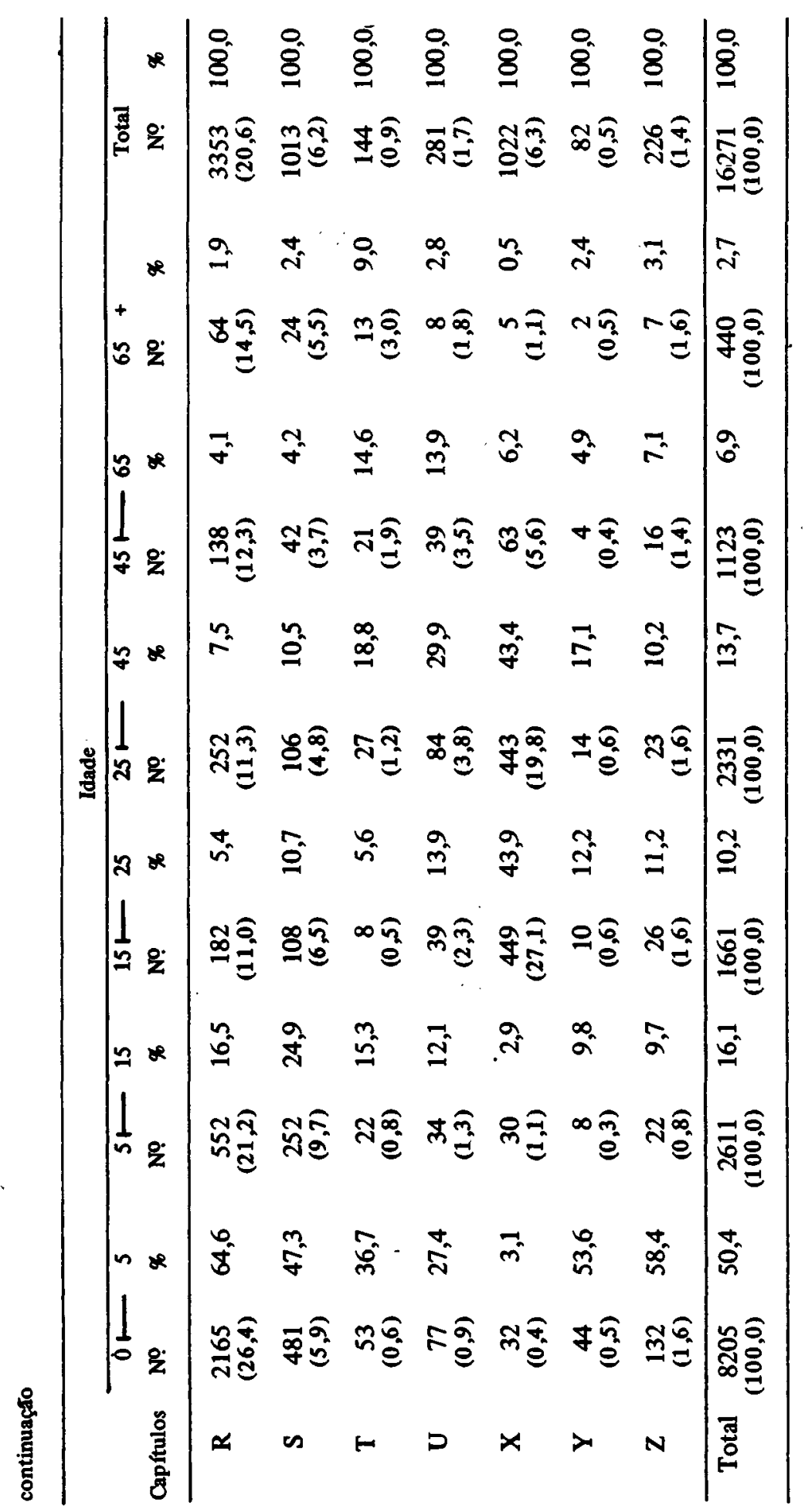


LEBRÃO, M.L. Classiflcaç İo internacional de motivos de consulta para assisténcia primária: teste em algumas áreas brasileiras. Rev. Satide pribl., S. Paulo, 19:69-78, 1985.

LEBRĀO, M.L. [An international classification of the reasons for seeling primary care]. Rev. Saúde públ., S. Paulo, 19: 69-78, 1985.

ABSTRACT: A new International Classification of Primary Care, proposed by WHO, is discussed. Comments are made on a field test carried out to investigate the feasibility of thus describing and characterizing the context' of primary care in Brazil. The results were satisfactory, leading to the conclusion that the classification is applicable.

UNITERMS: International classification of primary care.

\section{REFERENCIAS BIBLIOGRAFICAS}

1. CLASSIFICAÇĀO de Motivos de Consulta. São Paulo, Centro Brasileiro de Classificação de Doenças, 1983.

2. ICHPPC-2-Defined: international classification of health problems in primary care. $3^{\text {rd }}$ ed. Oxford, Oxford University Press, 1983.

3. MANUAL da Classificação Internacional de Doenças, Lesðes e Causas de Obitos, 9a revisão, 1975. São Paulo, Centro da OMS para Classificação de Doenças em Português/Ministério da Saúde/Organização $\mathrm{Pa}$ namericana da Saúde, 1980.2v.

4. MEADS, S. La classificacion de la OMS por "razones de la consulta". Cron. Org. mund. Salud, 37: 191-6, 1983.
5. NAPCRG1: a Process Code for Primary Care; International field trial version. Richmond, VA, North American Primary Care Research Group, 1981.

6. SCHNEIDER, D. An ambulatory classification system: design development and evaluation, Hlth Serv. Res., 14:77-8, 1979.

7. SCHNEIDER, D. et al. A reason for visit classification for ambulatory care. Vital Hith Statist. Ser. 2 (78) 1979.

8. WORLD HEALTH ORGANIZATION, Reasons for contact with primary care servi. ces: a model classification. Geneva, 1980.

Recebido para publicação em 14/09/1984. Aprovado para publicacão em 17/12/1984. 